[28] Posur. Deutsche Landwirtschaftliche Presse, t. LX, p. 16, 1933.

[29] Kroon. Zeitschrift für Züchtung, B. 19, 441 à 445, 1930.

[30] Dassat. Annali di Tecnica Agraria, fase. I, 8e année, 1934.

[31] RICHARDSEN. Landwirtschaftliche Jahrbücher, 1916, eit. par Dassat. Annali di Tecnica Agraria, fase. I, 8e année, 1934.

[32] Eckles et Williams. Journal of Dairy Science, VII, 1924 et VIII, 1925.

[33] Hart, Steienbock, KuINe et Humphrex. Journal of Biological Chemistry, t. LXXXVI, p. 145-155, 1930.

[34] LUcadon. Klinische Wochenschrift, no $5,1^{\mathrm{er}}$ février 1941.

\title{
ÉTUdE SUR L'EAU LIÉE DES FROMAGES
}

\section{par \\ G. MOCQUOT}

L'eau contenu dans les fromages ne se comporte pas vis-à-vis des substances dissoutes, comme l'eau qui s'écoule avec le sérum d'égoưttáge.

En 1929, WATsoN [1] et [2] mesure le point de congélation du fromage par la méthode cryoseopique et note des différences qu'il attribue à l'existence d'une proportion variable d'eau liée.

Un peu plus tard, MacDowall et Dolby [3], dans une étude sur la fabrieation du fromage Cheddar, ont dosé, au cours de l'égouttage, le lactosé dans le sérum, d'une part, et dans le caillé, d'autre part, et il's se sont aperęus que la concentration du sucre dans l'eau du caillé n'était pas la même que la concentration du sucre dans l'eau du sérum : la première est toujours inférieure à la seconde.

Les auteurs néo-zélandais en ont déduit qu'une certaine fraction de l'eau contenue dans le fromage devait être considérée comme liée aux substances organiques du caillé (bound water). Un calcul simple leur a permis de fixer cette proportion d'eau liée à 20 ou $30 \%$ de la substance sèche du caillé.

Après eux, Pyenson et Dahle (4) aux Etats-Unis, Alleman (5), en Suisse, ont utilisé la méthode cryoscopique. Pyenson et DaHLE ont opéré sur le lait et le sérum (obtenu après coagulation par la présure ou les acides). La mesure de l'abaissement du point de congélation résultant de l'addition d'un poids connu de sucre permet de calculer la quantité d'eau dans laquelle ce sucre est dissous. En faisant la différence avec l'eau totale, déterminée par dessication à l'étude, on en déduit au moyen de la formule de Newton et Gortner, la proportion d'eau qui ne dissout pas de sucre, c'est-à-dire l'eau liéé.

Pxenson et Dahle [4] ont déterminé, par différence, la proportion d'eau liée des divers constituants du lait, caséine, albumine, membrane des globules gras, ete. Dans le lait, la caséine à elle seule retient $55 \%$ de l'eau liée. C'est done elle qui joue dans le phénomène le rôle principal.

Allieman opère sur une émulsion de fromage, broyé dans l'eau distillée et détermine l'eau liée en utilisant la même formule quie PXENson et DAHLE. Ses résultats sont analogues à ceux des auteurs américains. Il a montré que dans les fromages à pâte dure, les parties voisines de la croûte, qui sont en général plus sèches, paraissent fixer moins d'eau d'hydratation. 
D'autre part, les substances azotées solubles et insolubles retiennent la presque totalité de l'eau d'hydratation, la matière grasse n'en retient qu'une quantité très faible.

Le sucre utilisé pour les déterminations eryoscopiques a toujours été du saccharose.

Les résultats obtenus par Pyenson et DARLE ainsi que ceux d'Allemann sont en assez bon accord avec les chiffres de MAcDowall et Dolby.

Dans le présent travail, nous avons opéré sur les caillés de fromagerie, selon une technique analogue à celle de MacDowall et Dolby. Des expériences préliminaires ont eu pour but de vérifier sur le gruyère les résultats obtenus par ces auteurs sur le Cheddar.

Nous avons cherché ensuite à déterminer l'influence du corps choisi comme indicateur sur la valeur trouvée pour le taux d'hydratation.

Enfin, nous avons étudié l'action sur l'eau liée des diftérents facteurs utilisés dans la pratique fromagère.

\section{EXPÉRIENCES PRÉLIMINAIRES}

Nous avons employé la méthode de MacDowall et DolBx [3] en l'appliquant au cas du fromage de gruyère, et en suivant la technique utilisée par ces auteurs au cours de leurs expériences sur le Cheddar.

Pendant la fabrication du gruyère, jusqu'au moment de la mise sous presse, la transformation du lactose en acide lactique est peuimportante, en raison de la température élevée et de la durée relativement brève du travail en chaudière.

Les prélèvements du caillé et du sérum n'offrent pas de difficultés et la comparaison des teneur en lactose de l'un et de l'autre se trouve indiquée dans le tableau I. Le calcul de la proportion d'eau liée est effectué en utilisant la formule de MacDowall [3]

$$
\text { Eau liée }=\frac{\mathrm{Ls}-\mathrm{Lc}}{\mathrm{Ls}} \times \frac{\mathrm{H}}{100 \cdot \mathrm{H}}
$$

dans laquelle $\mathrm{Ls}=$ concentration du lactose dans l'eau du sérum

$\mathrm{Lc}=$ concentration du lactose dans l'eau du caillé

$\mathrm{H}=$ teneur en ean du caillé.

Nos essaiv ont porté, soit sur du fromage gras $(45 \%$ de matière grasse dans la substance sèche), soit sur du fromage maigre, obtenu à partir du lait écrémé.

Nous avons fait figurer également dans ce tableau les résultats des dosages d'acide lactique dans le fromage sortant de presse (et ne contenant pratiquement plus de sucre).

La teneur en acide lactique $\%$ de fromage correspond assez exactement à celle que l'on peut calculer en partant du sucre contenu dans le caillé au moment de la mise sous presse, si l'on 
TABLEAU I

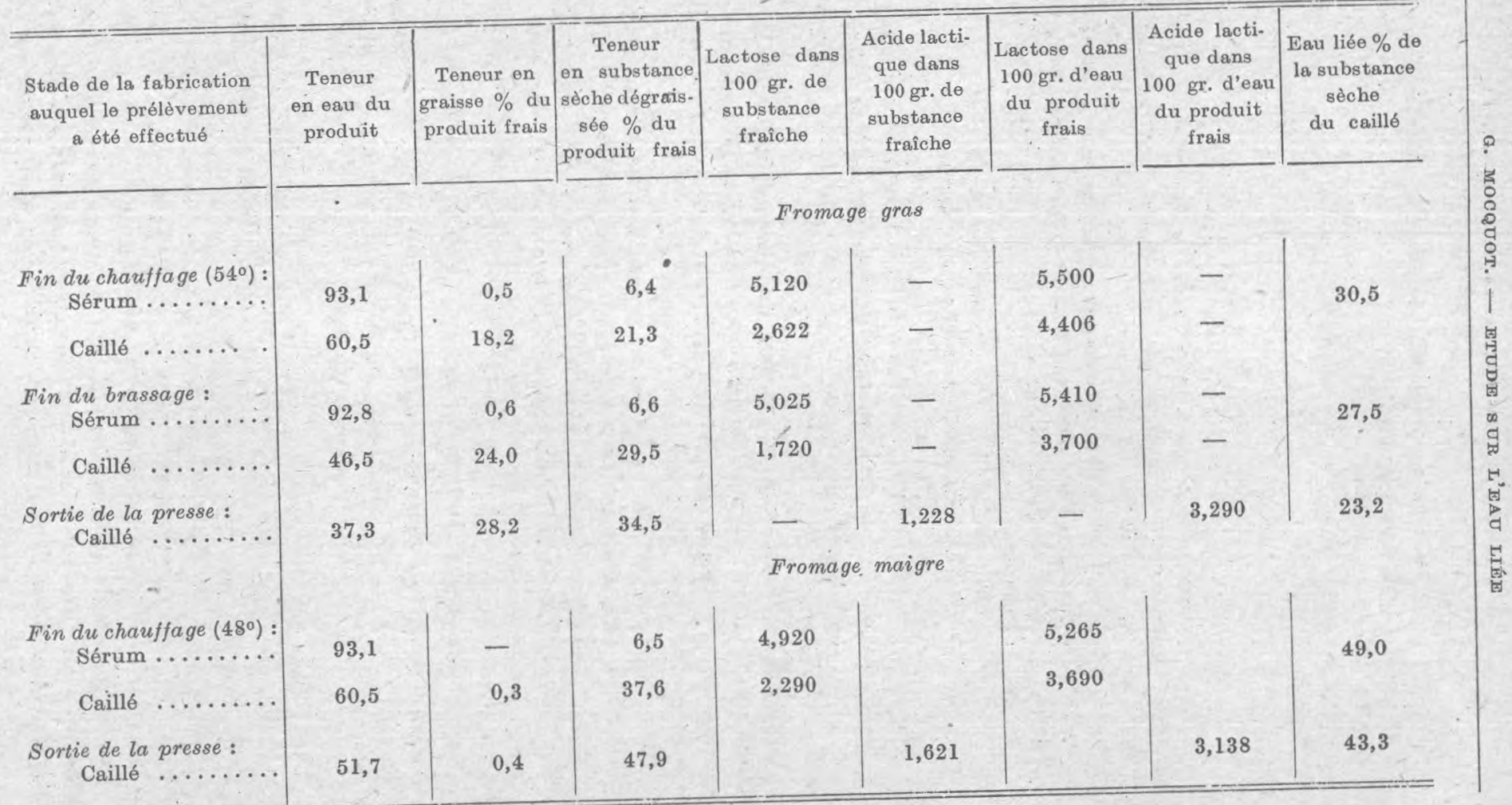


admet qu'un gramme de lactose (hydraté) donne un gramme d'acide lactique.

Cette comparaison suppose, en outre, que l'on ramène les résultats à des fromages de même humidité.

D'autre part, la presque totalité de l'azote se trouve, dans le fromage frais, sous forme insoluble de paracaséinate de calcium. C'est évidemment ce composé qui joue le rôle principal dans l'eau d'hydratation du fromage, car les chiffres obtenus pour les fromages gras et maigres indiquent nettement que la graisse n'a qu'une influence très faible.

C'est donc le comportement du paracaséinate de calcium que nous avons étudié plus spécialement dans la seconde série d'essais que nous allons décrire maintenant.

\section{DEUXIÈME SÉRIE D'EXPÉRIENCES}

\section{MÉTHODE DE DÉTERMINATION \\ DE L'EAU D'HYDRATATION}

DANS LES CAILLÉS DE FROMAGERIE

\section{Préparation des caillés}

Nous avons suivi, à l'échelle du laboratoire, un procédé de fabrication analogue à celui utilisé par les fromagers de gruyère en opérant de la façon suivante :

2 à 3 litres de lait écrémé frais, contenus dans une bassine, sont additionnés d'une quantité de présure convenable pour obtenir la coagulation en 15 ou 20 minutes à $34^{\circ}$.

Puis le caillé, abandonné à lui-même, "durcit " pendant 5 minutes. L'opérateur le découpe avec un petit tranche-caillé en fragments de $0 \mathrm{~cm} .5$ à 1 centimètre de côté, et commence à chauffer progressivement la bassine, en maintenant l'agitation, jusqu'à ce que la température atteigne $48^{\circ}-50^{\circ}$.

Après quelques instants de brassage à cette température, le sérum dans lequel baignent les grains de caillé, est décanté et les grains rassemblés dans une mousseline, sont lavés une première fois à l'eau, pour éliminer la plus grande partie du sérum.

Après refroidissement, le caillé, qui, dans l'intervalle, s'est soudé en masse, est râpé (sur une râpe à fromage) en menus fragments de dimensions aussi régulières que possibles (passage au tamis de 2 millimètres).

\section{Lavage du caillé}

Le eaillé râpé est lavé à l'eau courante, pendant un temps suffisant pour éliminer tout le lactose, et en même temps les éléments solubles. 
Ce lavage est réalisé en plaçant le caillé dans une mousseline à mailles fines, et en l'immergeant dans un récipient où circule un courant d'eau.

Après cinq à six heures, le caillé ne contient plus de lactose, on termine le lavage par plusieurs rinçages à l'eau distillée.

\section{Mise en contact avec la soluton servant d'indicateur}

Cette solution est toujours préparée à l'avance et refroidie à une température inférieure à $10^{\circ}$ (pour empêcher les proliférations microbiennes).

200 à 300 grammes de caillé lavé sont alors placés dans un flacon à large ouverture de 1 litre de capacité environ et la solution sucrée est ajoutée en quantité suffisante pour recouvrir le caillé (300 à $400 \mathrm{~cm}^{3}$ ). Un excès de liquide est indispensable. Le flacon est bouché et agité. La durée de contact peut être très brève : des temps de contact échelonnés entre 5 minutes et 12 heures ont donné les mêmes résultats, l'état d'équilibre est done atteint rapidement.

A partir de ce stade, les opérations sont différentes suivant que la substance indicatrice est volatile ou non volatile. Nous examinerons d'abord :

\section{A. Le cas des substances non volatiles.}

\section{Séparation DU CaIllé et dE LA SOLUTion INDICATRICe}

Le liquide dans lequel baignent les grains de caillé est filtré, sur un filtre en papier plissé ; la filtration est immédiate et le sérum recueilli à peine louche. Le caillé, prélevé à la main, ou avec une cuillère, dans le récipient à large ouverture, est essoré entre des feuilles de papier buvard, et placé dans une capsule; l'essorage est une opération essentielle pour obtenir un produit sur lequel il soit possible d'effectuer plusieurs prises d'essai comparables : si le caillé est trop humide en surface, les dosages ne sont pas concordants. Avec un essorage convenable, au contraire, deux ou trois prises d'essai conduisent exactement au même chiffre, tant pour la teneur en sucre que pour le dosage de matière sèche, ou celui de l'azote. La teneur en eau des produits obtenus varie de 68 à $65 \%$ avec une moyenne de $67 \%$.

2. Prélèvement des f́chantillons destinés auX analyses

Les prélèvements destinés aux analyses doivent être faits très rapidement, pour éviter la dessication d'une partie de l'échantillon.

a) Les prises d'essai du caillé sont effectuées avec une cuillère ou une spatule: les échantillons destinés aux dosages du sucre, dont le poids varie dans nos essais entre 15 et 40 grammes; sont 
introduits dans des Erlenmeyer préalablement tarés, et pesés, sur un trébuchet sensible au décigramme. Le dosage de l'humidité porte sur 2 à 3 grammes de caillé, pesés à la balance de précision dans les capsules contenant du sable de Fontainebleau $(10$ aे 15 grammes). Le dosage d'azote est effectué sur des prises de 1 gramme environ ( 2 ou 3 échantillons pour chaque essai).

b) Le liquide obtenu après filtration est prélevé à l'aide d'une pipette de précision (de $10 \mathrm{~cm}^{3}, 15 \mathrm{~cm}^{3}, 20 \mathrm{~cm}^{3}, 25 \mathrm{~cm}^{3}$ ). On en pèse un volume connu qui servira à la détermination de la substance sèche. La même pipette est utilisée ensuite pour mesurer les prises d'essai destinées au dosage du sucre; celles-ci sont ensuite introduites dans des fioles erlenmeyer de $250 \mathrm{~cm}^{3}$.

Les fioles contenant le caillé ou le sérum reçoivent chacune un volume déterminé - en général $100 \mathrm{~cm}^{3}$, exactement mesurés d'eau distillée Ces fioles sont bouchées et agitées.

\section{Dosages}

\section{๔) Dosage de la substance indicatrice.}

Les fioles contenant le caillé et le sérum sont agitées pendant 5 minutes environ.

De nombreux essais nous ont permis de constater que l'état d'équilibre entre la solution sucrée contenue dans le caillé et l'eau extérieure est atteint en quelques minutes ( 1 ou 2 minutes). Lorsqu'on étudié l'influence d'une durée d'agitation plus longue on constate que les résultats demeurent eonstants pendant 5 heures environ (à une température voisine de $+10^{\circ}$ ), puis la quanrité de sucre par gramme de caillé diminue légèrement, indiquant sans doute une destruction du sucre par les microorganismes toujours présents.

Or cette destruction est plus rapide dans la dilution du a caillé " que dans la dilution du "sérum", probablement par suite de la présence dans le caillé lui-même de la majeure partie des mieroorganismes susceptibles de détruire le sucre; ceci entraînerait une erreur dans l'estimation de l'eau liée. On évite cette erreur en opérant dans les limites de temps convenables : moins de 5 heures après le prélèvement de l'échantillon.

L'équilibre établi, le contenu des fioles est filtré sur papierfiltre Laurent, et le filtrat sert au dosage. Ce liquide est parfois tout à fait limpide et le dosage peut être effectué directement, mais le plus souvent, il présente un louche dû à des traces de substances protéiques qu'il est préférable d'éliminer. Un procédé qui nous a donné satisfaction consiste à ajouter à $50 \mathrm{~cm}^{3}$ de la solution sucrée $10 \mathrm{~cm}^{3}$ de $\mathrm{SO}^{4} \mathrm{H}^{2}$ et $7 \mathrm{~cm}^{3} 5$ d'acide phosphotungstique à $10 \%$. Laisser reposer 30 minutes, et filtrer. La plupart des sueres 
ne sont pas modifiés par ce traitement, seul le galactose est altéré. Dans ce cas, le trouble dû aux substances protéiques, peut être éliminé par filtration sur amiante.

1. Dosage du sucre. Le dosage du sucre est effectué par la méthode classique de Bertrand [6] qui permet d'obtenir une précision de l'ordre de $0,5 \%$ entre plusieurs dosages lorsque ceux-ci portent sur des quantités de sucre de 60 à 80 milligrammes dans la prise d'essai.

Nous avons utilisé également la méthode de HAGEDORNHensen [7] au ferricyanure, dont le degré de précision est à peu près le même que celui de la méthode Bertrand et qui s'applique à des doses de sucre beaucoup plus faibles $(0 \mathrm{mgr} .2$ environ dans la prise d'essai).

2. Dosage du glycérol. Nous avons employé la méthode de Fleury et Fatome [8] avec laquelle on peut doser 10 à 25 milligrammes de glycérol à $0,5 \%$ près.

\section{ß) Dosage de L'humidité.}

Après un séjour de 12 à 15 heures à l'étuve à $100^{\circ}$, les capsules sont refroidies à l'exsiccateur et pesées. Une deuxième pesée, effectuée après un nouveau séjour de 2 heures à l'étuve permet de s'assurer que la dessication est complète si le poids de la capsule n'a pas varié de plus de 1 à 2 milligrammes.

La précision des dosages est de 0,2 à $0,5 \%$.

\section{$\gamma)$ Dosage de L'AZOTE.}

L'azote contenu dans l'échantillon de 1 gramme de caillé est dosé par la méthode Kjeldahl $\left(20 \mathrm{~cm}^{3}\right.$ de $\mathrm{SO}^{4} \mathrm{H}^{2}$, 1 gramme $\mathrm{SO}^{4} \mathrm{Cu}$, 10 grammes $\mathrm{SO}^{4} \mathrm{~K}^{2}$ ) l'attaque est généralement facile et très rapide.

La distillation de l'ammoniaque se fait sur la totalité ou sur une partie aliquote du produit de minéralisation.

La précision des dosages est de $0,5 \%$.

\section{B. Cas où Jes substances indicatrices sont volatiles.}

1. Séparation du caillé et du sérum,

\section{PRÉLÈVEMENT DES ÉCHANTHLLONS DESTINÉS AUX ANALYSES}

La technique suivie est celle préconisée par Nicloux [9 et 10] et nous nous contenterons de la rappeler ici succinctement.

On opère à température assez basse $\left[10^{\circ}\right]$ moins pour éviter les proliférations microbiennes que pour empêcher toute évaporation de la substance indicatrice.

Le «liquide extérieur» est mesuré à la pipette et pesé dans un pèse-filtre bouché émeri. La plupart du temps, ce liquide ne con- 
tient que des traces très faibles de substances azotées (poussières de petits grains de caillé). La centrifugation peut permettre, si

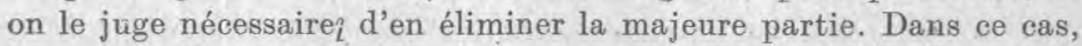
le plus simple consiste à mettre le caillé et le liquide en contact dans un tube de centrifugeuse bouché et à prélever, après centrifugation, le liquide clair dans la partie supérieure du tube.

Le caillé est prélevé avec une cuillère ou une spatule et pesé dans un autre pèse-filtre. L'essorage n'est pas nécessaire, puisque le dosage d'humidité est effectué sur le même échantillon qui sert au dosage d'alcool.

Le contenu de ehaque pèse-filtre est alors transvasé quantitativement dans un ballon de $500 \mathrm{~cm}^{3}$, renfermant déjà un certain volume d'eau froide. Le ballon est adapté à l'appareil de distillation de Schlosing-Aubin.

\section{Dosage}

a) De l'alcool et de l'acétone. La distillation est conduite de manière à recueillir environ $1 / 4$ du liquide à distiller. Le distillat obtenu renferme pratiquement tout l'alcool, ou l'acétone, contenu dans l'échantillon.

Le dosage est effectué, pour l'alcool, suivant la méthode de Nrcloux [11], pour l'acétone suivant la méthode de LindenBERG [12].

b) Dosage de la substance sèche. Après la distillation, le contenu du ballon est transvasé quantitativement dans une capsule tarée et sert au dosage de la matière sèche.

\section{Calcul des résultats}

Si l'on compare la concentration du sucre dans l'eau totale du caillé à la concentration du sucre dans le liquide extérieur, on constate que la première est inférieure à la seconde.

Reprenant alors l'hypothèse de NicLoux [9], et celle de MACDowaLl [3] nous exprimons les résultats en supposant qu'une fraction de l'eau liée (aux substances organiques) ne dissout pas de sucre; c'est leau liée de Nicloux, "the bound water" de MACDowaLl.

Dans l'autre fraction de l'eau (free water - eau libre) le sucre se trouve dissous à la même concentration que dans le liquide extérieur.

\section{A. Cas des substances non volatiles.}

Le calcul qui suit a pour but de permettre la détermination de la proportion d'eau liée, en tenant compte des résultats obtenus 
pour les dosages de sucre ou de glycérol, d'eau, de substance sèche et de substance azotée.

La figure I donne une représentation graphique du phénomène : il figure la répartition des divers éléments, eau, sucre, substance sèche, contenus dans les fioles Erlenmeyer.
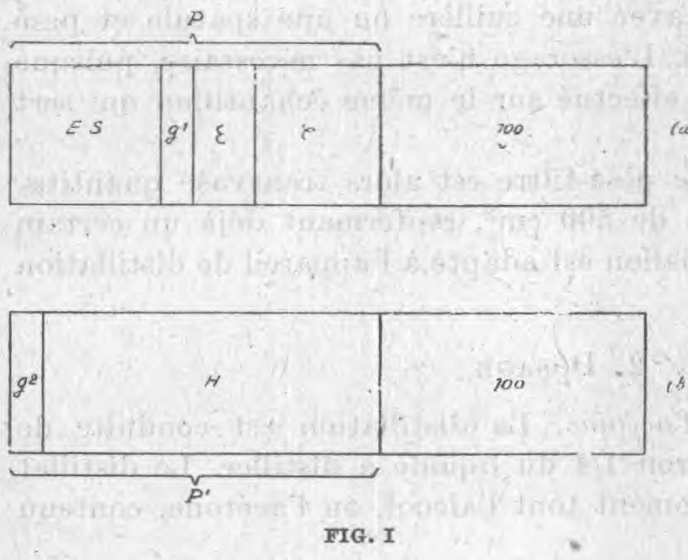

a) Représente le caillé.

La substance sèche (a) totale se décompose en :

E. S. D. = la substance sèche provenant du caillé dans la, prise d'essai.

$\mathrm{g}_{1}=$ le sucre (ou le glycérol) contenu dans la prise d'essai.

L'eau totale se décompose en :

$\varepsilon=$ eau liée (à la substance sèche du caillé).

$\varphi=$ eau libre.

Enfin, les 100 gr. d'eau ajoutés viendront diluer le sucre dissous dans l'eau libre.

b) se rapporte au "liquide extérieur» :

$\mathrm{g}_{2}=$ sucre contenu dans la prise d'essai.

$\mathrm{H}=$ eau totale de la prise d'essai.

On voit que : $\mathrm{g}_{2}+\mathrm{H}$ est pratiquement égal au poids $\mathrm{P}^{\prime}$ pesé à la balance.

Désignons par g la quantité de sucre par gramme de dilution : eau libre du caillé $+100 \mathrm{gr}$. eau + sucre $=\varphi+100+\mathrm{g}_{1}$.

Désignons par g' la quantité de sucre par gramme de la dilution : eau du sérum + sucre $+100 \mathrm{gr}$. eau $=\mathrm{P}^{\prime}+100$.

$\mathrm{g}$ et $\mathrm{g}^{\prime}$ représentent les résultats des dosages tels qu'ils sont fournis par l'analyse.

Exprimons la concentration $\mathrm{C}_{1}$ du sucre dans l'eau libre du caillé, avant la dilution par les 100 grammes d'eau,

$$
\mathrm{C}_{1}=\frac{\mathrm{g}_{1}}{\varphi+g_{1}}
$$


d'où l'on peut direr la valeur de

$$
\varphi=\frac{g_{1}\left(1-C_{1}\right)}{C_{1}}
$$

D'autre part, après l'addition des 100 grammes d'eau, on a

$$
\mathrm{g}_{1}=\mathrm{g}\left(100+\varphi+\mathrm{g}_{1}\right)
$$

d'où :

$$
g_{1}=\frac{g(100+\varphi)}{1-g}
$$

en remplaçant $g_{1}$ par cette expression dans l'équation (2) on obtient pour $\varphi$ la valeur suivante :

$$
\varphi=\frac{\log g\left(1-G_{1}\right)}{C_{1}-g}
$$

Ie schéma $(b)$ indique la valeur $\mathrm{deC}_{1}=\frac{\mathrm{g}_{2}}{\mathrm{P}^{\prime}}=\frac{\mathrm{g}^{\prime}\left(100+\mathrm{P}^{\prime}\right)}{\mathrm{P}^{\prime}}$

en remplaçant $C_{1}$ par sa valeur dans l'expression (5), on obtient la formule permettant de connaître $\varphi$ en fonction de $g, g^{\prime}$, et $\mathrm{P}^{\prime}$ :

$$
\varphi=\frac{100 \mathrm{~g}\left[\mathrm{P}^{\prime}\left(1-\mathrm{g}^{\prime}\right)-100 \mathrm{~g}^{\prime}\right]}{\mathrm{g}^{\prime}\left(100+\mathrm{P}^{\prime}\right)-\mathrm{g} \mathrm{P}^{\prime}}
$$

Le dosage d'humidité nous indique, d'autre part, la valeur de l'eau totale $H$ contenue dans la prise d'essai. L'eau liée $\varepsilon$ est donc égale à :

$$
\varepsilon=\mathrm{H}-\varphi
$$

On peut exprimer $\varepsilon$ en fonction de la substance sèche du eaillé, après déduction du sucre contenu dans ce dernier, on a en effet :

E $\mathrm{S}=$ substance sèche totale

$-\mathrm{g}_{1}=$ sucre du caillé

$\mathrm{ESD}=$ substance sèche provenant du caillé.

On peut enfin exprimer $\varepsilon$ en fonction de la matière azotée, dont le pourcentage dans le caillé humide a été déterminé.

\section{B. Cas des substances indicatrices volatiles.}

Nous avons calculé la proportion d'eau liée d'après la formule de Nicloux [9] :

$$
K=\frac{100(1-\varphi)}{8}-1
$$


dans laquelle $\mathrm{K}=$ quantité d'eau liée pour 1 gramme de substance sèche.

$$
P=\frac{Q a}{Q e} \text { dans laquelle }\left\{\begin{array}{c}
\mathrm{Qa}=\begin{array}{c}
\text { teneur en alcool ou acétone du } \\
\text { caillé } ;
\end{array} \\
\mathrm{Qe}=\text { teneur en alcool ou acétone du } \\
\text { liquide. }
\end{array}\right.
$$

Lorsque le sérum contient lui aussi une certaine quantité de substance sèche, Nicloux a montré que la formule devenait :

$$
K=\frac{100(1-\rho)}{s_{1}-\rho_{2}}\left\{\begin{array}{l}
s_{1}=\text { substance sèche du caillé. } \\
s_{2}=\text { substance sèche du sérum. }
\end{array}\right.
$$

\section{Les résultats :}

1. Vartations de La teneur en eaU Lí́e SUIVANT LA SUBSTANCE UTILISÉE COMME "INDICATEUR》

L'examen du tableau 2 résumant les essais effectués avec les différentes substances non électrolytes dont nous nous sommes servis comme indicateurs, révèle des différences marquées :

Celle qui sépare, par exemple, les sucres en C 6 des sucres en C 12 et du raffinose en C 18 est intéressante à noter. Les trois sucres en C 6 (glucose, lévulose, galactose) donnent des chiffres voisins les uns des autres, de même avec les trois sucres en C 12 (saccharose, maltose, lactose) on obtient des valeurs du même ordre, mais nettement supérieures aux précédentes. Enfin, avec le raffinose, le résultat est plus élevé que pour tous les autres.

La différence qui sépare les sucres en C 6 des sucres en $\mathrm{C} 12$ a été vérifiée très simplement de la façon suivante : on prépare une solution de saccharose que l'on divise en deux portions.

La première est mise en contact avec le caillé directement et la proportion d'eau liée trouvée par l'expérience est alors égale à $40 \%$ de la substance sèche.

La seconde est préalablement invertie par $\mathrm{HCl}$ et neutralisée, puis mise en contact avec le caillé, la détermination d'eau liée donne le chiffre de $25 \%$ de la substance sèche. Nous avons vérifié que cette variation ne peut être imputêe, dans les conditions où nous nous sommes placés, à la présence de $\mathrm{NaCl}$ (résultant de la neutralisation par la soude ou le carbonate de soude du liquide d'inversion). On en déduit que la scission de la molécule de saccharose a modifié son comportement vis-à-vis de l'eau du fromage.

Si l'on passe aux substances de poids moléculaires plus faibles : l'arabinose en C 5, l'aldéhyde glycérique, le glycérol donnent à peu près la même valeur d'eau liée que les sucres en C 6 . 
TABLEAU 2

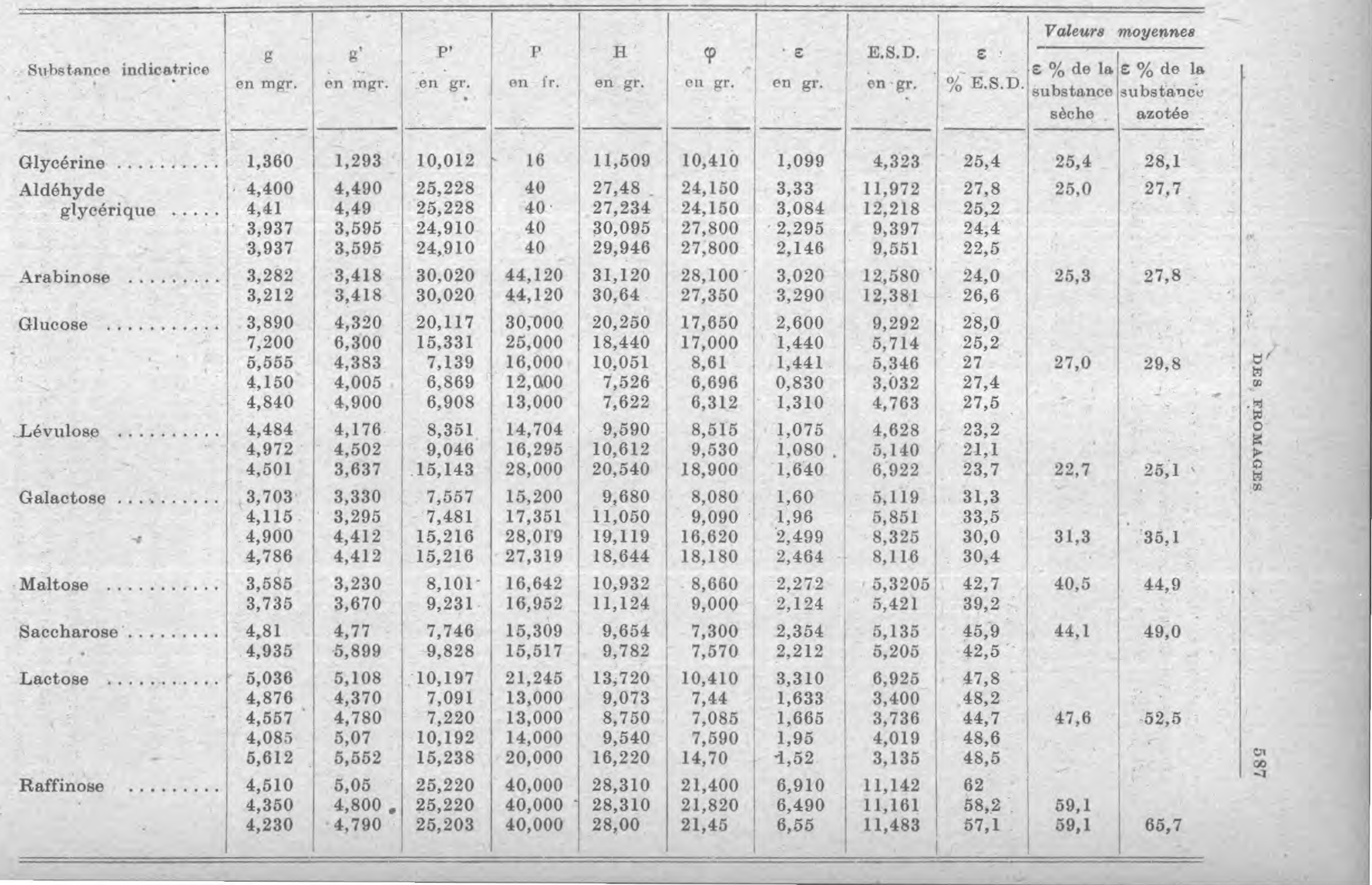


Par contre, en employant l'acétone et surtout l'alcool éthylique nous avons trouvé des chiffres plus bas. Pour l'alcool éthylique la proportion d'eau liée est très faible ou nulle (tableau 3).

TABLEAU 3

\begin{tabular}{|c|c|c|c|c|c|c|c|}
\hline $\begin{array}{l}\text { Substance indi- } \\
\text { catrice }\end{array}$ & $\begin{array}{l}\text { Durée du } \\
\text { contact entre le } \\
\text { caillé et la solu- } \\
\text { tion acétonique }\end{array}$ & Qa & Qe & & $\mathrm{s}_{1}$ & $p^{8}{ }_{2}$ & Eau liée \\
\hline \multirow[t]{4}{*}{ Acétone } & 30 minutes & 0,3365 & 0,3696 & 0,9125 & 9,90 & 0,32 & 17,5 \\
\hline & 2 heures & 0,1682 & 0,2061 & 0,816 & 15,40 & 0,02 & 19,7 \\
\hline & 24 heures & 1,129 & 1,369 & 0,826 & 16,10 & 0,426 & 11,5 \\
\hline & 5 jours & 1,128 & 1,406 & 0,802 & 16,72 & 0,544 & 22,5 \\
\hline \multicolumn{8}{|l|}{ Aleool éthy } \\
\hline lique $\ldots \ldots$ & *15 heures & 1,424 & 1,931 & 0,737 & 30,5 & 5,18 & 4,0 \\
\hline & *15 heures & 1,406 & 1,924 & 0,731 & 32,21 & 5,29 & 0,0 \\
\hline & 15 heures & 1,143 & 1,719 & 0,667 & 33,78 & 0,2535 & 0,5 \\
\hline & 15 heures & 1,250 & 1,719 & 0,728 & 28,64 & 0,276 & 3,0 \\
\hline
\end{tabular}

Les essais indiqués* ont porté sur des grains de caillé non lavés, en suspension dans le lactesérum, d'où la teneur en substance sèche plus élevée du sérum et la valeur qui en découle pour le produit $\mathrm{ps}_{2}$.

\section{Variation de la teneur en eaU lí́E}

SOUS L'ACTION DES FACTEURS MIS EN JEU DANS LA PRATIQUE FROMAGEेRE

a) Influence $d u$ chauffage.

Le lait qui doit servir à la préparation du caillé est réparti entre deux récipients $\mathrm{A}$ et $\mathrm{B}$.

L'addition de présure et le découpage du caillé sont conduits de façon identique pour $A$ et $B$ alors que le chauffage est différent.

A est chauffé à $35^{\circ}$ seulement.

B est chauffé à $50^{\circ}$.

Les opérations ultérieures sont les mêmes qu'à l'ordinaire.

Les résultats sont consignés dans le tableau 3. On constate, dans le cas du chauffage à $50^{\circ}$, une légère diminution de la proportion d'eau liée, une diminution beaucoup plus importante de l'eau "libre".

Les variations de la température de chauffage dépassent évidemment celles que l'on rencontre dans la pratique usuelle, mais comme la teneur en eau liée ne change pas beaucoup, même dans le cas de ces températures extrêmes, on en déduit que les 
variations de plus faible amplitude nè peuvent avoir qu'une influence encore moins marquée.

\section{b) Influence de la pression.}

Nous avons cherché quelle pouvait être l'action de ce facteur en modifiant les conditions de l'essorage (se reporter à la technique décrite ci-dessus). Avant d'être essoré, le caillé est divisé en deux portions; la première subit un essorage léger, juste suffisant pour permettre d'effectuer des prises d'essai homogènes, la seconde est fortement essorée et pressée.

Les résultats ressemblent à ceux que nous avons obtenus à propos de l'action de la température; une forte pression diminue peu le pourcentage d'eau liée, mais abaisse dans une proportion beaucoup plus grande la teneur en eau totale.

\section{c) Influence du salage.}

Après broyage et tamisage, le caillé est réparti en deux lots, - le premier est mis en contact avec une solution sucrée témoin, - le second est mis en contact avec une solution sucrée et salée (20\% de sel marin).

La suite des opérations est la même que dans le procédé habituel : on dose en outre le taux des chlorures dans le liquide extérieur et dans le caillé.

Les résultats sont consignés dans le tableau 4.

TABLEAU 4

\begin{tabular}{|c|c|c|}
\hline Nature de l'essai & $\begin{array}{l}\text { Substance sèche } \% \text { de } \\
\text { la substance humide } \\
\text { (sucre déduit) }\end{array}$ & $\begin{array}{c}\text { Eau liée } \\
\% \text { de la substance sèche } \\
\text { (sucre déduit) }\end{array}$ \\
\hline Pression : & Lactose & Glucose \\
\hline ............ & 29,9 & $28,8 \quad 46,6$ \\
\hline Forte $\ldots \ldots \ldots \ldots \ldots$ & $36,1 \quad 33,0$ & $24,6 \quad 45,3$ \\
\hline $\begin{array}{l}\text { Chauffage : } \\
\text { A } \mathbf{3 5 0} \ldots \ldots \ldots \ldots \ldots\end{array}$ & 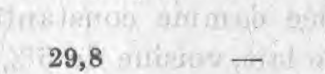 & $\mathbf{3 2 , 3}+6) ; y$ \\
\hline A $509 \ldots$ & 38,5 & 8nsint \\
\hline Salage : & 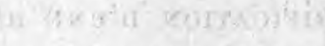 & \\
\hline Nul ........... & 30,0 & $27,5 \quad 45,6$ \\
\hline A $20 \%$ de sel $\ldots$. & 32,55 & $19,5 \quad 30,5$ \\
\hline
\end{tabular}

On a déduit de la substance sèche le suére et le sel qu'elle contient. 
On voit que le salage à nettement diminué la proportion d'eau liée et que cette diminution affecte à peu près également les sucres en C 6 et les sucres en C 12 : elle représente une perte d'eau liée d'environ $30 \%$ par rapport aux taux primitifs. Par contre, l'ean totale n'a pas beaucoup varié.

Il est intéressant de noter le changement des propriétés physiques du caillé plongé dans la saumure salée. Ce dernier perd en effet son élasticité et durcit beaucoup. Ces phénomènes sont bien connus des fromagers qui utilisent le sel pour obtenir l'égouttage des fromages à pâtes persillées (type Roquefort) ou du Port-Salut.

L'action particulière du sel sur l'eau liée diffère, par conséquent de celle du chauffage et de la pression qui diminuent surtout l'eau "libre» et atteignent moins l'eau liée.

La comparaison des teneurs en eau libre qui serait intéressante pour mesurer le degré de déshydratation auquel on est parvenu, est délicate, car il est difficile d'effectuer, sur de petites quantités, toutes les opérations de préparation du caillé, en particulier, l'essorage, dans des conditions rigoureusement semblables, de sorte que l'humidité finale du caillé utilisé est toujours quelque peu variable, sans que ces variations puissent être attribuées de façon certaine à l'influence de tel ou tel facteur.

\section{InFluence de LA CONCENTRATION DU SUCRE EMPloý́}

La concentration des solutions sucrées utilisées varie de 6 à $1,5 \%$, dans le liquide extérieur, sans que les proportions d'eau liée subissent de changementš notables.

\section{TABLEAU 5}

Concentrations en sucre :

$2 \% \quad 6 \%$

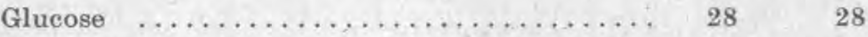

Galactose ........................ $30 \quad 31$

Lévulose ........................ $23 \quad 22$

Lactose $\ldots \ldots \ldots \ldots \ldots \ldots \ldots \ldots \ldots \ldots \ldots \ldots \ldots \ldots 4474$

Il semble que, dans les limites envisagées, on puisse considérer la proportion d'eau liée comme constante. La concentration normale du lactose dans le lait, voisine de $5 \%$, se trouve comprise entre ces limites.

4. VÉRIFICATION D'UNE HYYPOTHÈSE

\section{D'UNE EaU LIÉE a la SUBSTANCe ORganique dU CaIllé}

Une cause d'erreur pourrait s'introduire dans nos résultats si l'on supposait qu'une certaine fraction de la substance indicatrice restait fixée sur le caillé soit au moment de l'addition de $100 \mathrm{~cm}^{3}$ 
d'eau distillée (cas des substances non volatiles), soit pendant la distillation (cas des substances volatiles).

Les expériences effectuées par NıcLoux permettent d'éliminer cette hypothèse dans le cas des substances volatiles; pour les substances non volatiles, nous avons vérifié (en nous plaçant dans le cas des sucres) que cette fixation ne se produit pas et que l'interprétation du phénomène décrit est correcte. Voici notre essai :

On prépare une certaine quantité de caillé suivant la méthode exposée ci-dessus. Après lavage, ce caillé est essoré, entre deux feuilles de papier buvard, et deux échantillons sont prélevés. Le permier sert à la détermination de l'humidité, le second échantillon, de poids $\mathrm{P}$, est mis en contact avec un poids $\mathrm{P}^{\prime}$ de solution sucrée dont on connaît la teneur en sucre C 1.

La quantité de sucre contenue dans le poids $\mathrm{P}^{\prime}$ de solution est égale à : $\mathrm{P}^{\prime} \times \mathrm{C}_{1}$.

L'eau du caillé vient diluer cette solution sucrée et après obtention d'un état d'équilibre (d'ailleurs rapidement atteint) on trouve que la concentration du sucre a diminué dans la solution et est devenue $\mathrm{C}_{2}$.

Si l'on appelle x la quantité d'eau contenue dans la nouvelle solution, on a

$$
\mathrm{C}_{2}=\frac{\mathrm{P}^{\prime} \mathrm{C}_{1}}{\mathrm{x}+\mathrm{P}^{\prime} \mathrm{C}_{1}}
$$

d'où l'on tire $\mathrm{x}=\frac{\mathrm{P}^{\prime} \mathrm{C}_{1}\left(\mathrm{I}-\mathrm{C}_{2}\right)}{\mathrm{C}_{2}}$

D'autre part, l'eau " totale» est égale à l'eau de la solution sucrée, à laquelle s'ajoute l'eau du caillé, ce qui peut s'écrire :

$$
\lambda=\mathrm{P}^{\prime}\left(1-\mathrm{C}_{1}\right)+\mathrm{P} \times \mathrm{H}
$$

La différence $\lambda-x$ représente l'eau qui n'a pas dissous de sucre, c'est l'eau liée que l'on peut rapporter à la substance sèche du caillé $(100-H) \mathrm{P}$.

A titre d'exemple, voici les résultats obtenus dans l'une de nos expériences.

La solution sucrée utilisée contenait du glucose. Pour que le caillé soit bien immergé dans cette solution et que le contact puisse s'établir facilement on pèse un poids de caillé :

dont l'humidité :

$$
\mathrm{P}=70 \mathrm{gr} \text {. }
$$

$$
H=65,6
$$

et un poids de solution sucrée :

$$
\mathrm{P}^{\prime}=165 \text { gr. } 200
$$


dont la concentration en sucre :

$$
\begin{gathered}
\mathrm{C}_{1}=0 \text { gr. } 01395 \text { par gramme } \\
\mathrm{P}^{\prime} \mathrm{C}_{1}-165,2 \times 0,01395=2 \mathrm{gr} .310
\end{gathered}
$$

D'autre part, la concentration en sucre du mélange final (solution sucrée + caillé) $\mathrm{C}_{2}=0$ gr. 01131 par' gramme d'où :

$$
\begin{aligned}
\mathrm{x}=\frac{\mathrm{P}^{\prime} \mathrm{C}_{1}\left(\mathrm{l}-\mathrm{C}_{2}\right)}{\mathrm{C}_{2}} & =\frac{2,31(1-0,01131)}{0,01131} \\
& =201,5 .
\end{aligned}
$$

Enfin, la quantité d'eau totale (eau du caillé + eau de la solution sucrée)

$$
\begin{aligned}
\lambda & =\mathrm{P} \times \mathrm{H}+\mathrm{P}^{\prime}\left(1-\mathrm{C}_{1}\right) \\
& =65,6 \times 70+(165,2-2,31)=208 \text { gr. } 84 .
\end{aligned}
$$

La différence $\lambda-x=208,84-201,5=7,34$.

L'eau liée est égale à $30,5 \%$ de la substance sèche du caillé.

Remarque. La différence entre les deux chiffres $\lambda$ et $s$ est assez faible; exprimés en pour cent de $\lambda$ elle est voisine de $3 \%$ alors que dans la méthode employée habituellement, la différence entre l'eau totale et l'eau libre est exprimée par 45,95-38,61 =7,34 où l'eau liée représente $14 \%$ de l'eau totale.

La seconde méthode décrite dans le paragraphe ci-dessus n'est donc pas à conseiller comme méthode d'investigation mais sa précision est cependant suffisante pour démontrer que les résultats obtenus par le premier procédé ne doivent pas être imputés à une fixation du sucre par le caillé.

Discussion. En accord avec les résultats obtenus par d'autres auteurs, nous avons montré qu'une certaine proportion de l'eau retenue dans les caillés de fromagerie se comporte comme si elle ne dissolvait pas les sucres ou d'autres substances qui nous ont servi dans cette étude. L'hypothèse d'une eau "liée» nous a permis de calculer le nombre de grammes d'eau retenue par gramme de substance seche du eaillé (sucre déduit).

Mais cette, hypothèse n'est probablement pas la seule que f'on puisse faire : celle d'une solubilité variable des substances indicatrices dans diverses fractions de l'eau du caillé aboutirait aux mêmes conclusions. Le phénomène serait alors progressif au lieu d'être discontinu.

Les différences qui séparent des corps de poids moléculaires variables montrent en tous cas qu'il ne peut être question de définir une seule "eau liée" : il convient encore de préciser qu'il s agit de l'eau liée mise en évidence au moyen d'une substance indicatrice déterminee. 
A cet égard, il est intéressant de noter qu'à notre connaissance, les chiffres cités par les différents auteurs qui ont étudié l'eau liée des constituants du lait ou des fromages ont été obtenus en employant comme "indicateur" un sucre en C 12 (saccharose ou lactose) qu'il s'agisse de la méthode cryoscopique ou de celle qui utilise la mesure des différences de concentration. Ceci explique la similitude de leurs résultats.

Après avoir montré que les différents sucres "pénètrent" chacun à leur manière dans l'eau du caillé, il est intéressant de savoir ce qui se produirait si le lactose du lait venait à être remplacé par du glucose, en imaginant par exemple que le sucre du sang puisse passer, sans transformation, dans le lait secrété par la mamelle.

En pareil cas, le taux du sucre dans l'eau du fromage et par suite dans le fromage lui-même, serait augmenté puisque le glucose pénètre dans une fraction de l'eau du caillé qui reste "imperméable" au lactose.

Le taux d'acide lactique provenant de la fermentation de ce sucre par les bactéries lactiques, subirait un accroissement analogue. Or on connaît l'importance du taux d'acide lactique dans les fromages, et les conséquences d'une augmentation ou d'une diminution éventuelle de ce dernier [13]. Dans le fromage de gruyère, une augmentation du taux d'acide lactique conduit au défaut désigné par les pratiFromage normal (Gruyère) - Le sucre du lait est du ciens sous le nom de "lailactose.

100 grammes de fromage contiennent : nure »: la pâte se fend au lieu de produire une ouverture normale.

On peut prévoir qu'un lait dans lequel le lactose serait remplacé par du glucose donnerait après transformation une forte proportion des fromages lainés, les conditions de fabrication demeurant par ailleurs inchangées.

La figure II ci-contre donne une idée de l'augmentation du taux d'acide lactique dans un "fromage au glucose " comparé à celui qui existe dans un fromage normal. Extrait dégraissé
mon azoté dont
8r. 68 d'acide lactique 
D'autre part, le phénomène que nous avons rencontré en opérant sur le caillé de fromagerie présente une certaine analogie avec celui déjà décrit par Polonovski et LINDENBERG [14].

Ces auteurs opèrent tout d'abord sur le goujon placé dans de l'eau additionnée de substances non électrolytes de poids moléculaires variables; alcool méthylique, alcool isopropylique, acétone, méthyl-éthyl-cétone.

Ils calculent le rapport B/A, dans lequel :

$\mathrm{A}=$ teneur en alcool ou cétone pour mille de l'eau extérieure.

B = teneur en alcool ou cétone pour mille de l'eau des tissus.

Ils constatent que ce rapport est inférieur à l'unité et s'en écarte d'autant plus que la substance ajoutée possède un poíds moléculaire plus élevé.

C'est ce que nous avons trouvé nous-même avec les sucres et les autres substances employées au cours du présent travail.

En étudiant les poissons vivant dans l'eau de mer, PoLonovsikr et LINDENBERG ont vu que le rapport B/A était sensiblement égal à l'unité, et qu'il devenait supérieur à 1 lorsque les poissons étaient placés dans une eau de mer à double concentration.

Les essais de salage du caillé ne nous ont pas permis d'arriver à un résultat semblable, et nous a vions seulement constaté, dans le cas des sucres, un abaissement de la teneur en eau liée, sous l'influence du sel. D'autres expériences seraient done nécessaires pour s'assurer de la similitude des deux phénomènes et nous poursuivons nos recherches sur ce point.

\section{Résumé}

Une première série d'essais nous a permis de mettre en évidence, dans le cas du fromage de gruyère, l'existence d'une certaine proportion d'eau liée, phénomène déjà signalé par MACDowALl et DoLBY, dans leurs travaux sur la fabrication du Cheddar.

Dans une deuxième série d'expériences, nous avons comparé, après élimination du lactose par lavage du caillé; la concentration de différentes substanees non électrolytes dans l'eau du eaillé et dans l'eau extérieure.

Une relation entre le poids moléculaire des substances en diffusion et la proportion d'eau liée a été établie. L'influence des divers facteurs mis en jeu dans la pratique fromagère (pression, chauffage, salage) a fait l'objet d'une dernière série d'essais. La pression et le chauffage ne modifient guère la proportion d'eau liée, le salage a une action plus marquée.

\section{BIBLIOGRAPHIE}

[1] P. D. WATSON. Jour. of Dairy Sci., vol X, p. 331, 1927.

[2] P. D. Watson. Jour. of Dairy Sci., vol. XII, 1929.

[3] MacDowall et DolBy, Jour. of Dairy Research, vol. VIII, no 2, p. 156, 1936. 
[4] Pyenson et C. D. Dahle. Jour. of Dairy Sci., vol. XXI, p. 169, 407, $601,1938$.

[5] O. Almemann. Annuaire Agric, de la Suisse, 90 fasc., p. 936, 1940.

[6] G. Brtrand. Bull. soc. Chim., (3) 35, 1285, 1906.

[7] HaGedorn-Jensen, Biochem. Zeitschr., 135, 46, 137, 92, 1923.

[9] Fleury et Fatome. Ann. des fermaetations, 36, t. I, p. 285, 1935.

[8] Nicloux. Bull. Soc. Chim. Biol., 16, p. 788, 1934.

[10] Nichoux. Travaux des membres de la Soc. de Chim. Biol., 25, p. 1046, 1943.

[11] Nicloux. Annales des fermentations, 36, t. I, p. 449, 1935.

[12] Linden Berg. Compte rendu de la Soc. de Biol., t. II, p. 317, 1936.

[13] R. M. Dolby, H. F. MacDowali et K. K. R. MacDowhll. Jour. of Dairy Research, vol. VIII, no 1. 0. 74, 1937.

[14] Polonovski et Lindenberg. Bull. Soc. Chim. Biol., 10, p. 1323, 1938.

(Manuscrit recu pour publication en Aout 1946).

\title{
REVLE
}

\section{L'INDUSTRIE LAITIÊRE A L'ÉTRANGER}

\author{
par \\ G. GENIN \\ Ingénieur E.P.C.I.
}

\section{ALLEMAGNE}

\section{Le barattage continu du beurre}

De nouvelles informations ont été publiées concernant les procédés de fabrication continue du beurre qui ont été mis au point au cours de la guerre en Allemagne et qui permettent d'accélérer la fabrication, de réduire les dépenses de main-d'cuvre, de diminuer les pertes de matière grasse et d'obtenir un beurre de qualité améliorée.

Dans un des procédés dénommé procédé Fritz, on utilise une baratte spéciale ayant une capacité de production de près de 500 kilogrammes par heure. On introduit dans cet appareil la cxème renfermant 40 à $45 \%$ de graisse et maintenue à une température de 6 à $8^{\circ}$. Cette crème pénètre dans un cylindre horizontal d'environ 250 millimètres de diamètre, refroidi à l'eau, qui tourne à une très grande vitesse et qui transforme la crème en beurre. Le mélange de granules de beurre et de petit-lait est alors séparé dans une autre chambre, grâce à l'action de deux vis de 40 centimètres de longueur environ, de 15 centimètre de diamètre et qui tournent on sens opposé à la vitesse de 40 tours par minute. A la sortie de cette chambre, le beurre est obligé de passer dans une 